\title{
Editorial
}

\section{Tonsillectomies, ketorolac, and the march of progress}

It is an old adage in medicine that "the rarer the complication, the more likely it is to result in a change in policy." Is the issue of postoperative bleeding after a nonsteroidal anti-inflammatory drug (NSAID), such as ketorolac, an example of this cliché? Or, is ketorolac an agent that is contraindicated in children undergoing tonsillectomy because of demonstrated risk of haemorrhage?

The NSAIDs have been an interesting addition to our armamentarium for pain relief in children. Although they are primarily peripherally acting, they can have powerful effects on inflammatory mediators and, consequently, primary and secondary hyperalgesia at a peripheral level.' Several studies have demonstrated that ketorolac is an effective analgesic for a variety of procedures. Effective analgesia has been demonstrated for procedures that are associated with mild (but real) postoperative pain, such as myringotomies, ${ }^{2}$ as well as more painful procedures such as strabismus repair, ${ }^{3}$ tonsillectomies, ${ }^{4}$ and orthopaedic procedures. ${ }^{5}$ There are reputed benefits of considerably less nausea and vomiting, sedation, or respiratory depression when compared with opioids. ${ }^{1,5}$ However, NSAIDs have some troubling side effects, with reported instances of decreased bone repair after osteotomy, ${ }^{6}$ acute renal failure, ${ }^{7}$ and bronchospasm.

In 1992, Hannallah commented on postoperative analgesia in the paediatric patient in an editorial in this journal. In that editorial, the "clinical significance of the drug's effect on platelet function must be evaluated objectively in a large number of patients before its routine use in children who are prone to postoperative haemorrhage, such as following tonsillectomy, can be recommended." This continues to be a concern in the anaesthesia community and is the subject of a paper in this issue. ${ }^{9}$

From the Department of Pediatric Anesthesia, Children's Memorial Hospital, 2300 Children's Plaza, Chicago, Ill 60614 , U.S.A.
The potential for NSAIDs to promote increased bleeding is based on their known capability of altering platelet function. Of historical interest, Singer published a paper in 1945 cautioning practitioners about the potential of aspirin for promoting extraordinary haemorrhage after tonsillectomy. ${ }^{10}$ The effect of NSAIDs on isolated platelet function, as well as more global measures of coagulation, has been extensively studied in awake, normal patients. ${ }^{11,12}$ There are differences between NSAIDs in their ability to inhibit platelet function, based on varying degrees of inhibition of platelet cyclo-oxygenase activity." There are several studies on ketorolac's activity, with most demonstrating a modest prolongation of bleeding time, decreased production of thromboxane and prolonged aggregation in response to collagen, ${ }^{13}$ but no significant alteration in thromboelastography, aggregation in response to adenosine, platelet count, or prothrombin/partial thromboplastin times. ${ }^{1,14,15}$ The most consistent finding has been an increase in bleeding time, but the clinical importance of this test has been questioned. ${ }^{16}$

The ability of ketorolac to inhibit platelet cyclo-oxygenase activity may be balanced, in part, by an increase in platelet function related to the stress of a surgical procedure. Platelet aggregation has been shown to be accelerated after surgical stress, possibly secondary to stimulation of alpha ${ }_{2}$-adrenergic receptors on the platelet membrane. ${ }^{17}$ Thwaites demonstrated, in adults, that although intravenous ketorolac use was associated with almost complete inhibition of platelet cyclo-oxygenase activity, measurements of platelet aggregation, as well as bleeding time and thromboelastography, were not affected. ${ }^{18}$ The importance of the balance between platelet function inhibition from ketorolac and function stimulation from the surgical procedure is not well quantitated in the literature. The ability of surgical stress should not be counted on to prevent the possibility of bleeding, given the many studies indicating an increased bleeding in clinical situations.

The drug circular states that "Toradol ${ }^{\circledR}$ is "contraindicated" as a prophylactic analgesic before any 
major surgery and is CONTRAINDICATED intraoperatively when hemostasis is critical because of the increased risk of bleeding." (Drug circular, HoffmannLa Roche Inc., 1995) The use of the word "contraindicated" is, of course, a powerful admonition by the manufacturer to not use this drug in any situation where platelet function inhibition could result in haemorrhage. Is tonsillectomy a surgical procedure for which ketorolac is contraindicated?

Splinter's article is one of several recent studies in the anaesthesia literature specifically examining the role of ketorolac in children undergoing tonsillectomy. Unlike the many adult studies that focus particularly on orthopaedic cases, the paediatric tonsillectomy/adenoidectomy is a special concern. Not only are these common procedures, but they can be associated with considerable blood loss, with an incidence of postoperative haemorrhage estimated to be between 2 and $5 \% .{ }^{19}$ Although surgeons make efforts to establish adequate haemostasis, the literature demonstrates a consistent incidence of postoperative bleeding, occasionally necessitating surgical reexploration.

Of interest, Rusy, ${ }^{20}$ Gunter, ${ }^{21}$ and Gallagher ${ }^{19}$ found an increased incidence of postoperative haemorrhage in patients who received ketorolac, as did Splinter. However, in all except Gunter's study, the ketorolac was given before the surgical procedure was finished. In contrast, Sutters did not find increased bleeding when the ketorolac was given at the end of the procedure. ${ }^{22}$ The concern that this practice and design raises is whether the timing of drug administration is a crucial issue. If ketorolac is given before adequate hemostasis is achieved, it is possible that ketorolac-induced platelet dysfunction will interrupt the normal clotting sequence, subsequently causing an increase in postoperative blood loss. In both Gallagher and Splinter's series, not only was there an increase in intraoperative bleeding, but an increased incidence of patients returning to the operating room for reexploration. In Rusy's study, there was increased difficulty in establishing haemostasis intraoperatively, but no patients required reexploration for bleeding.

The study by Gunter et al. is particularly disturbing because of a demonstrated incidence of increased bleeding even though the ketorolac was given after surgical haemostasis was established. The study was actually terminated prematurely because of concern about the increased incidence of bleeding episodes in the first 24 $\mathrm{hr}$ after surgery. Although there was a higher incidence of bleeding episodes in the first $24 \mathrm{hr}$ with the ketorolac group, the total number of patients experiencing postoperative bleeding was not different between the two groups. It has been suggested by Gunter (personal com- munication) that the increased bleeding in the first $24 \mathrm{hr}$ is an indication that the surgical site after a tonsillectomy is different than other wounds in that there is ongoing loss and reformation of clot due to movement of the area, making it particularly vulnerable to decreased clotting ability even after initial haemostasis is obtained. However, the overall incidence of bleeding in both the ketorolac and opioid groups was $16 \%$, an unexpectedly high rate that may be an indication of the small sample size. Does this article prove that ketorolac given after surgical haemostasis is achieved promotes bleeding? The small sample size is, in my opinion, inadequate to draw that conclusion.

The desirability of a non-opioid for postoperative analgesia is attractive, especially in a patient population that may be at risk for airway obstruction, as well as nausea and vomiting. Ketorolac initially was enthusiastically tried by anaesthetists because of its potent analgesic properties. However, clinical experience and the sudden appearance of studies indicating increased haemorrhage has dissuaded many practitioners, both anaesthetists and surgeons, from using ketorolac specifically for tonsillectomies.

This has become an emotional issue for many practitioners, both among those who use the drug and those who eschew it. The literature, including the current article, indicates that there is reason for concern. On the other hand, there continues to be considerable use of ketorolac by many practitioners. It is of interest that, with the exception of Splinter's article, the rest of the quoted prospective studies in tonsillectomy patients contain less than a hundred subjects each, with the drug usually given before surgery is finished. It is possible that excessive bleeding is not a problem if ketorolac is only given after haemostasis has been established, but this will require additional studies to prove. To some extent, we have not progressed far from Dr. Hannallah's concerns in 1992 - the clinical significance of altered platelet reactivity remains to be established in large numbers of patients. 


\section{L'amygdalectomie, le kétorolac et la marche du progrès}

En médecine, il existe un vieil adage qui énonce qu'une complication, par sa rareté même, est susceptible de provoquer un changement d'attitude. Cet adage s'appliquerait-il à l'hémorragie postopératoire consécutive à l'administration d'une anti-inflammatoire non stéroüdien (AINS) comme le kétorolac? Ou encore, le kétorolac serait-il contre-indiqué chez des enfants qui subissent une amygdalectomie parce que des preuves l'associent à un risque accru d'hémorragie?

Les AINS représentent des ajouts importants aux agents dont nous disposons. Bien qu'ils agissent avant tout en périphérie, il peuvent avoir de puissants effets sur les médiateurs de l'inflammation et, par conséquent, provoquer à ce niveau de l'hyperalgie primaire et secondaire.' Plusieurs études ont démontré l'efficacité du kétorolac comme analgésique dans une grande variété d'interventions. La preuve de cette efficacité est faite pour les douleurs postopératoires légères (mais réelles), comme celles de la myringotomie, ${ }^{2}$ ou plus importantes comme celles de la correction de strabisme, ${ }^{3}$ de l'amygdalectomie, ${ }^{4}$ et de certaines interventions orthopédiques. ${ }^{5}$ Lorsqu'on les compare aux morphiniques, on reconnaît aussi aux AINS des bénéficies: en effet, ils réduisent les nausées et les vomissements, la sédation et la dépression respiratoire. ${ }^{1-5}$ Cependant les AINS ont des effets secondaires inquiétants, tels le ralentissement de la restauration osseuse après une ostéotomie, ${ }^{7,6}$ l'insuffisance rénale aiguë ${ }^{7}$ et le bronchospasme.

En 1992, dans éditorial publié dans ce journal, Hannallah émettait le commentaire suivant: "l'importance en clinique des effets du médicament sur la fonction des plaquettes doit subir une évaluation objective chez un grand nombre de patients avant qu'on puisse recommander son utilisation courante chez les enfants sujets à l'hémorragie postopératoire comme celle qui suit l'amygdalectomie ${ }^{8}{ }^{8} \mathrm{Ce}$ danger continue de préoccuper le monde de l'anesthésie et est maintenant le sujet d'un article. ${ }^{9}$

Il est reconnu que les AINS exagèrent le saignement parce qu'il sont capable d'entraver le fonctionnement des plaquettes. Du point de vue historique, il est intéressant de noter que Singer a publié en 1945 un article signalant aux clinicients que l'aspirine pouvait provoquer des hémorragies exagérées après l'amygdalectomie. ${ }^{10}$
L'influence des AINS sur la fonction des plaquettes isolées comme sur les autres mesures de la coagulation a été étudiée à profusion chez le patient normal et éveillé. ${ }^{11,12}$ La capacité d'inhibition de la fonction plaquettaire n'est pas la même pour tous les AINS et relève du degré d'inhibition de l'activité de la cyclo-oxygénase plaquettaire." Il existe plusieurs études portant sur l'activité du kétorolac qui, pour la plupart, révèlent une prolongation modeste du temps de saignement, une diminution de la production de la thromboxane, une prolongation de l'agrégation en présence de collagène ${ }^{13}$ mais pas d'altérations importantes du thromboélastogramme, de prolongation de l'agrégation en présence d'adénosine, de la numération des plaquettes ou des temps de prothrombine et de céphaline. ${ }^{14.15}$ La prolongation du temps de saignement est la caractéristique la plus fréquemment notée mais on ne s'entend pas sur l'importance de cette épreuve. ${ }^{16}$

La capacité d'inhibition par le kétorolac de la cyclooxygénase plaquettaire peut être contrebalancée en partie par une augmentation de la fonction plaquettaire causée par le stress chirurgical. Il a été démontré que le stress chirurgical accélérait l'agrégation des plaquettes, possiblement par stimulation des récepteurs $\alpha_{2}$-adrénergiques situés sur la membrane plaquettaire. ${ }^{17}$ Chez l'adulte, Thwaites a montré que bien que le kétorolac intraveineux soit associé à une inhibition presque complète de l'activité de la cyclo-oxygénase plaquettaire, les mesures de l'agrégation plaquettaire, du temps de saignement et de la thromboélastographie n'étaient pas affectées. ${ }^{18}$ Dans la littérature, l'importance de l'équilibre entre l'inhibiton par le kétorolac de la fonction plaquettaire et sa stimulation provoquée par l'intervention chirurgicale n'est pas bien quantifiée. Étant donné le grand nombre d'études faisant état d'une augmentation du saignement dans des situations cliniques, il ne faut certes pas compter sur l'intervention du stress chirurgical pour prévenir l'hémorragie.

La circulaire qui accompagne le médicament signale que « le Toradol ${ }^{\circledR}$ est contre-indiqué comme analgésique prophylactique avant toute chirurgie majeure quand l'hémostase est en péril à cause du risque accru de saignement " (circulaire, Hoffmann-LaRoche Inc., 1995). L'utilisation du mot contre-indiqué par le manufacturier constitue une recommandation convaincante d'éviter ce produit dans les situations où l'inhibition de la fonction plaquettaire pourrait favoriser l'hémorragie. L'amygdalectomie fait-elle partie des interventions pour lesquelles le kétorolac est contre-indiqué?

L'ćlude de Splinter est, en anesthésie, une étude parmi plusieurs qui spécifiquement considèrent le rôle du kétorolac hez les enfants qui doivent subir une amygdalectomic. Contrairement aux études chez l'adulte qui 
s'intéressent particulièrement aux interventions orthopédiques, le cas de l'amygdalectomie/adénoïdectomie suscite une préoccupation particulière. Non seulement, il $s$ 'agit là d'interventions très fréquentes, mais elles peuvent être associées à une incidence d'hémorragies de l'ordre de 2 à $5 \% .{ }^{20}$ Malgré toute la rigueur de l'hémostase chirurgicale, la littérature révèle la persistance de l'hémorragie postopératoire qui nécessite parfois une révision en salle d'opération.

Il est intéressant de noter que Rusy, ${ }^{20}$ Gunter $^{21}$ et Gallagher, ${ }^{19}$ ont trouvé, comme Splinter, chez les patients sous kétorolac, une incidence accrue du saignement postopératoire. Cependant, chez tous à l'exception de Gunter, le kétorolac était administré avant la fin de l'intervention. Par contre, Sutters, n'a pas rencontré d'aggravation du saignement lorsque le kétorolac était administré à la toute fin de l'intervention. ${ }^{22}$ On peut donc se demander si, selon cette façon de procéder, le moment de l'administration du kétorolac ne constituerait pas le noeud du problème. Si le kétorolac est administré avant la réalisation d'une hémostase suffisante, on peut supposer que le kétorolac provoque un dysfonctionnement des plaquettes qui interrompt la cascade normale de la coagulation et augmente en conséquence le saignement. Tant dans la série de Gallagher que dans celle de Splinter, on constatait non seulement une augmentation du saignement peropératoire mais aussi une augmentation du nombre des sujets qui devaient retourner en salle d'opération pour une révision. Dans l'étude de Rusy, l'hémostase peropératoire était plus difficile à établir, mais aucun des patients n'a dû retourner en salle d'opération à cause d'un saignement.

L'étude de Gunter et al. était particulièrement troublante parce qu'elle révélait une augmentation du saignement même si le kétorolac avait été administré une fois l'hémostase complétée. L'étude avait même été arrêtée prématurément à cause de l'inquiétude causée par une augmentation des épisodes de saignement pendant les premières $24 \mathrm{~h}$ postopératoires. Bien que l'incidences des épisodes de saignement dans les premières $24 \mathrm{~h}$ postoperératoires ait augmenté dans le groupe kétorolac, le nombre total des patients sujets à l'hémorragie postopératoire n'était pas différent entre les deux groupes. Gunter a suggéré (communication personnelle) que l'augmentation du saignement des premières $24 \mathrm{~h}$ démontre que le site chirurgical de l'amygdalectomie est différent de celui des autres plaies en ce que les manipulations endommagent un caillot qu'il doit être constamment régénéré, ce qui le rend particulièrement vulnérable à la baisse de la capacité de coaguler même une fois l'hémostase établie. Cependant, l'incidence totale de saignement était de $16 \%$, un taux étonnamment élevé qui pourrait s'expliquer par la petite dimension de l'échantillon. Cet article prouve-t-il que le kétorolac administré après l'hémostase chirurgicale favorise le saignement? La dimension de l'échantillon est, à mon avis, insuffisant pour tirer cette conclusion.

Pour l'analgé postopératoire, les avantages d'une médication non morphinique sont attrayants surtout pour une population à risque d'obstruction respiratoire ainsi que de nausées et de vomissements. Initialement le kétorolac a été mis à l'épreuve avec enthousiasme par les anesthésistes à cause de ses propriété analgésiques. Cependant, l'expérience acquise et l'apparition rapide d'études mettant en évidence une augmentation de l'hémorragie ont dissuadé nombre de praticiens, tant anesthésistes que chirurgiens, d'utiliser spécifiquement le kétorolac pour l'amygdalectomie.

C'est maintenant dans un climat chargé d'émotivité que plusieurs praticiens s'affrontent, ceux qui utilisent le produit et ceux qui le rejettent. La littérature, incluant l'article actuel, montre qu'on a raison de s'inquiéter. Cependant, plusieurs continuent de l'utiliser à profusion. Il est intéressant de noter, qu'à l'exception de l'article de Splinter, le reste des études prospectives citées concernant l'amygdalectomie contient moins de cent sujets chacune et que le produit est ordinairement administré avant la fin de la chirurgie. Il est possible que le kétorolac ne cause pas de problèmes si on l'administre une fois l'hémostase établie, mais des études additionnelles sont nécessaires pour le prouver. Nous n'avons pas tellement fait de progrès depuis l'énoncé du docteur Hannalah en 1992 - chez un grand nombre de patients, la signification clinique de l'altération de la réactivité plaquettaire demeure à établir.

\section{References}

1 Souter AJ, Fredman B, White PF. Controversies in the perioperative use of nonsteroidal antiinflammatory drugs. Anesth Analg 1994; 79: 1178-90.

2 Watcha MF, Ramirez-Ruiz $M$, White $P F$, Jones $M B$, Lagueruela $R G$, Terkonda RP. Perioperative effects of oral ketorolac and acctaminophen in children undergoing bilatcral myringotomy. Can J Anaesth 1992; 39: 649-54.

3 Mendel HG, Guarnieri KM, Sund LM, Torjman MC. The effects of ketorolac and fentanyl on postoperative vomiting and analgesic requirements in children undergoing strabismus surgery. Anesth Analg 1995; 80: 1129-33.

4 Marher SJ, Peutrell JM. Postoperative morphine requirements, nausea and vomiting following anaesthesia for tonsillectomy. Comparison of intravenous morphine and nonopioid analgesic techniques. Paediatric Anaesthesia 1995; 5: $185-8$.

5 Fragen RJ, Stulberg SD, Wixson R, Glisson S, Librojo E. Effect of ketorolac tromethamine on bleeding and on 
requirements for analgesia after total knee arthroscopy. Bone Joint Surg Am 1995; 77: 998-1002.

6 Ho ML, Chang JK, Wang G.J. Antiinflammatory drug effects on bone repair and remodeling in rabbits. Clin Orthop 1995; 313: 270-8.

7 Haragsim L, Dalal R, Bagga H, Bastani B. Ketorolacinduced acute renal failure and hyperkalemia: report of three cases. Am J Kidney Dis 1994; 24: 578-80.

8 Hannallah RS. Postoperative analgesia in the paediatric patient (Editorial). Can J Anaesth 1992; 39: 641-2.

9 Splinter WM, Rhine EJ, Roberts DW, Reid CW, MacNeill $H B$. Ketorolac tromethamine increases bleeding after tonsillectomy in children. Can J Anaesth 1996; 43: 560-3.

10 Singer $R$. Acetylsalicylic acid, a probable cause for secondary post-tonsillectomy hemorrhage. Arch Otolaryngol Head Neck Surg 1945; 42: 19-20.

11 Dordoni $P L$, Della Ventura $M$, Stefanelli A, et al. Effect of ketorolac, ketoprofen and nefopam on platelet function. Anaesthesia 1994; 49: 1046-9.

12 Conrad KA, Fagan TC, Mackie MJ, Mayshar PV. Effects of ketorolac tromethamine on hemostasis in volunteers.

Clin Pharmacol Ther 1988; 43: 542-6.

13 Pallapies D, Peskar BA, Brune K, Geisslinger G. Effects on platelet functions and pharmacokinetics of azapropazone and ketorolac tromethamine given as single parenteral doses. Br J Clin Pharmacol 1994; 37: 335-9.

14 Gibbs NM, Sear JW. Effect of ketorolac, bupivacaine and low-dose heparin on thromboelastographic variables in vitro. Br J Anaesth 1995; 75: 27-30.

15 Reinhart DJ, Latson TW, Whitten CW, Klein KW, Allison $P M$, Patel $M$. Influence of ketorolac tromethamine on clot elastic strength in humans as assessed by thromboelastography. J Clin Anesth 1993; 5: 216-20.

16 Lind SE. The bleeding time does not predict surgical bleeding. Blood 1991; 77: 2547-52.

17 Rosenfeld BA, Faraday N, Campbell D, et al. Perioperative platelet reactivity and the effects of clonidine. Anesthesiology 1993; 79: 255-61.

18 Thwaites BK, Nigus DB, Bouska GW, Mongan PD, Ayala $E F$, Merrill $G A$. Intravenous ketorolac tromethamine does not worsen platelet function during knee arthroscopy under general anesthesia. Anesth Analg 1995; 81 : 119-24.

19 Gallagher JE, Blauth J, Fornadley JA. Perioperative ketorolac tromethamine and postoperative hemorrhage in cases of tonsillectomy and adenoidectomy. Laryngoscope 1995; 105: 606-9.

20 Rusy LM, Houck CS, Sullivan LJ, et al. A double-blind evaluation of ketorolac tromethamine versus acetaminophen in pediatric tonsillectomy: analgesia and bleeding. Anesth Analg 1995; 80: 226-9.

21 Gunter JB, Varughese AM, Harrington JF, et al. Recovery and complications after tonsillectomy in chil- dren: a comparison of ketorolac and morphine. Anesth Analg 1995; 81: 1136-41.

22 Sutters $K A$, Levine JD, Dibble S, Savedra M, Miaskowski $C$. Analgesic efficacy and safety of single-dose intramuscular ketorolac for postoperative pain management in children following tonsillectomy. Pain 1995; 61: 145-53. 